\title{
Psycho-oncologie des cancers cérébraux
}

\author{
M.-F. Bacqué \\ (C) Springer-Verlag France 2010
}

\section{Une « très » mauvaise nouvelle}

« Oui, il s'agit bien d'une tumeur. Oui, cette tumeur est bien une tumeur primitive du cerveau. Oui, comme nous pouvions le redouter sur les images initiales, et comme nous en avions parlé, cette tumeur correspond à une tumeur maligne, à un cancer du cerveau ». Langage de vérité, paroles ciselées qui tentent d'amener à la réalité tout en s'apprêtant à accueillir les réactions les plus extrêmes. Le redoutable est bien là, et il trône au cœur même de l'organe noble qui définit aujourd'hui notre humanité. Mais ce cancer du cerveau est très loin de s'apparenter aux autres cancers d'organes, il est « le » cancer, celui qui effraie et qui trouble le plus. Nous voici à la conjonction de trois univers anxiogènes : le cancer du cerveau nous renvoie tous à la mort, à la folie et à la démence dégénérative. Trois dimensions dans lesquelles l'homme sombre à l'annonce du diagnostic : la mort anticipée dans l'imagerie sociale du cancer, la perte du partage avec autrui dans la folie, l'absence de commande de son propre corps et d'orientation dans le temps et l'espace avec la démence... Que reste-t-il alors ?

\section{Où est le patient ?}

C'est en effet la question la plus inquiétante. Les médecins et les équipes soignantes savent qu'ils sont confrontés à l'absence potentielle psychologique et morale du patient. Cet effacement progressif de la subjectivité qui transforme le dialogue habituel en une sourde perplexité du malade qui s'étonne, mais se résigne devant sa perte de familiarité avec lui-même... On pense aux patients déments qui se heurtent à leur propre incapacité à répondre à la question simple « où sommes-nous ? » et qui se remémorent leur lieu de vie privilégié, " mais à la maison... », tout en constatant intérieurement qu'il ne peut pas s'agir de cela, mais qu'ils ne disposent d'aucune autre réponse. On pense à Jacques Brel

\footnotetext{
M.-F. Bacqué ( $\square)$

Université de Strasbourg, département de psychologie,

12 , rue Goethe,

F-67000 Strasbourg, France

e-mail : mfbacque@club-internet.fr
}

«j'ai jamais tué de chat. Ou c'était il y a longtemps... Ou y sentaient pas bon »... Hélas, ici, pas d'excuse, les neurones ont foutu le camp...

\section{Des atteintes cérébrales secondaires en augmentation}

Pourtant, les tumeurs cérébrales primitives ne représentent que $2 \%$ des maladies cancéreuses, mais les métastases cérébrales sont les tumeurs malignes les plus fréquentes. Et leur incidence augmente du fait du vieillissement de la population, et surtout, paradoxalement, du fait de la survie prolongée des patients atteints de cancer.

Face à cette augmentation, les services de neuro-oncologie se développent et mettent au point des approches spécialisées. Elles concernent plusieurs domaines comme l'annonce, les traitements, la qualité de vie, l'évaluation neuropsychologique et son croisement avec les symptômes psychiatriques confondants. La neuro-oncologie pourrait aussi être pionnière dans la compréhension des réactions des proches face à la démentification précoce, l'impression de perte d'humanité et de la capacité de penser de celui qu'ils aimaient.

Beaucoup de sollicitations intimes pour ces médecins, infirmiers, assistants sociaux, psychologues qui aujourd'hui sont conscients des défenses qui auraient pu les conduire jadis à éviter la confrontation avec ces malades. Les neurologues ont été longtemps considérés comme les plus intellectuels des médecins, mais aussi les moins « affectifs». Un préjugé comme un autre qui s'appuyait sans doute sur cette attitude de recherche d'un diagnostic complexe, puis sur cet abandon devant l'absence d'issue thérapeutique. Aujourd'hui, les équipes pallient la difficulté à investir un patient dont le pronostic est sombre, en multipliant les possibilités thérapeutiques qui stimulent dorénavant la relation avec ces malades. Une relation authentique installée d'emblée a donc toutes les chances d'être pérenne pendant des années, ce qui est à l'origine d'un véritable investissement mutuel soignant-patient.

\section{Dire sans dire?}

L'équipe de l'unité de neuro-oncologie du CHU de Nancy, sous la plume de R. Lanfroy et al. a adapté cette consultation 
d'annonce si particulière quand il s'agit de « dire sans mentir, dire sans blesser et parfois dire sans dire $» .$. . Ce moment essentiel est partagé entre médecin, infirmière, personne de confiance et patient. Il n'est plus empreint de la solennité de jadis, mais se déroule dans un échange interactif naturel, puisque les acteurs se connaissent depuis le début de l'enquête. C'est ainsi que le ton juste est trouvé, que l'authenticité mêlée de respect accompagne l'ensemble de la révélation et qu'un véritable « contrat» d'humanité est passé entre tous. La justesse de cet « appareil » repose bien évidemment sur des personnes singulières, mais leurs qualités sont universelles et peuvent être retrouvées chez tous les professionnels.

L'idée que ce contrat initial perdure pendant l'ensemble du suivi, redonne confiance quand les malades s'aggravent. Car s'ils se sentent «étrangers à eux-mêmes », ils sont aussi perçus par leurs proches comme de plus en plus étranges, et ce doute réciproque engendre colère, tristesse, solitude. La notion de « soins continus » prend ici tout son sens.

\section{Qu'évaluer sur le plan neuropsychologique?}

La tendance à l'éclatement des professionnels, comme autant de spécialistes de fonctions variées, ne devrait pas être à l'origine de la demande de bilan neuropsychologique. Les psychologues qui ont cette formation clinique à l'étude de la dégradation des fonctions cognitives connaissent bien le risque de ne s'attacher qu'à des résultats partiels. Tous les coauteurs de ce numéro montrent que leur considération est avant tout globale. Les tests ne sont pas considérés uniquement pour leurs qualités psychométriques, mais ils prennent en compte les influences de l'humeur ou les représentations pessimistes du cancer sur les résultats. Cependant, on insistera sur la difficulté de compréhension de ces personnes, parfois aphasiques ou mutiques, le plus souvent déprimées ou dont les troubles résultent plutôt d'un chemo brain que des conséquences de la tumeur. À l'origine de ces déficits cognitifs, on retrouve aussi bien la fatigue que la dépression, les difficultés neurologiques réversibles que les handicaps définitifs. Ces patients ne sont souvent pas inclus dans les essais cliniques, ce qui a un impact sur les résultats statistiques des traitements, puisque seuls ceux qui vont le mieux (et qui peuvent s'exprimer) seraient évalués.

Pour les symptômes dépressifs, la situation n'est guère meilleure. Si le quart des patients présentent un épisode dépressif majeur, sa reconnaissance échappe à beaucoup d'équipes. Ainsi, après la chirurgie de la tumeur cérébrale, l'écart est énorme entre médecins qui constatent que $22 \%$ des patients sont déprimés, alors que $94 \%$ des malades s'auto-estiment comme déprimés trois mois après l'opération [1]. On remarquera que la maladie neuro-oncologique autant que les chimiothérapies entrainent de nombreux symptômes confondants : insomnie ou hypersomnie, agita- tion ou ralentissement psychomoteur, fatigue, anhédonie, diminution de la concentration et de l'aptitude à penser. Une corrélation entre les tests neuropsychologiques et la qualité de vie permet de les utiliser aussi pour évaluer cette dernière : en effet, la détérioration des tests précède celle de la qualité de vie.

Enfin, les tests neuropsychologiques permettent de mieux comprendre les effets des traitements et du cancer. Ainsi, l'irradiation cérébrale améliore les fonctions neuropsychologiques des patients parce qu'elle diminue les altérations physiques et psychologiques induites par les métastases. On a donc la preuve que la première cause de dégradation neuropsychologique est due à l'évolution des métastases.

Ces résultats peuvent convaincre et surtout rassurer les patients qui subissent une irradiation cérébrale. Outre, les différents accidents et leur impact médiatique (Épinal et Toulouse), la peur de la radiothérapie est encore intense. Le port d'un masque peut être angoissant, la solitude sous la machine, ou les rayons qui, bien qu'indolores, dégagent une impression de modernité ambiguë, enfin, les effets secondaires de l'irradiation de la tête entrainent gonflement et bouleversement des traits du visage qui atteignent l'identité. Pouvoir parler intimement et intégrer ces changements si abrupts de la représentation de soi est l'une des importantes possibilités données par les psychologues de ces services.

\section{Le plan cancer insiste sur la réadaptation sociale...}

Mais, seuls $20 \%$ des patients avec une tumeur cérébrale retrouveront un travail à temps plein... Et pour combien de temps ? Les cancers cérébraux posent bien la question du handicap d'origine centrale : comment faire quand on n'a plus d'équilibre, plus d'autonomie de sa mobilité, plus de capacités à communiquer ou tout simplement des aptitudes relationnelles très perturbées?

La rééducation cognitive est encore balbutiante et pourtant elle montre des résultats très encourageants. Des programmes avec des patients présentant un gliome de bas grade donnent une récupération importante de l'autonomie et des stratégies cognitives nécessaires à toute implication professionnelle ou sociale. Les outils d'évaluation de ces progrès existent, même s'il reste difficile de démêler les troubles de l'humeur, la fatigue ou les effets des traitements dans les causes de ces bouleversements.

Les tumeurs cérébrales créent donc des difficultés d'une grande complexité. Elles atteignent le plus noble, le plus central des organes. Elles blessent l'être humain dans ce qui fait son identité, sa personnalité, son désir d'être reconnu comme humain. Face à tant d'adversité, nous sommes heureux de constater que l'époque du traitement solitaire face à un neurologue gêné et glacial est révolue. La 
constitution d'équipes qui partagent une éthique humaniste permet au contraire d'aborder franchement et avec attention un diagnostic encore générateur d'effroi et de détresse. La place des neuropsychologues cliniciens a clairement démontré l'intérêt d'évaluer les difficultés cognitives, mais aussi affectives. Les thérapeutiques sont du même ordre, soutien psychologique, psychothérapies et rééducation cognitive, offrent au patient une relation approfondie dans laquelle il reste un sujet. Les neuro-oncologues, les chimiothérapeutes autant que les radiothérapeutes sont aujourd'hui autant préoccupés de durée de survie, que de qualité de vie. Ils ne sont plus seuls et agissent en synergie avec leurs collaborateurs psychosociaux. L'inclusion des proches n'est plus un obstacle et fournit au contraire autant d'occasions d'intégra- tion du sujet malade dans son groupe. La fin de vie peut alors y être mieux adaptée. Elle ne devrait plus constituer une terminaison fatale, mais elle pourrait être poursuivie par une réflexion des soignants sur la prise en charge globale des malades ou par un soutien des proches épuisés par leur accompagnement. Renverser la charge psychique éreintante de ces cancers en une plus grande humanisation des services de santé, tel est le pari tenu par ce numéro...

\section{Référence}

1. Litofsky N, Scott M, Farace E et al (2004) Depression in patients with high-grade glioma: results of the Glioma Outcomes Project. J Neurosurg 54:358-67 